\title{
Effect of uphill walking on browning factor and high molecular weight-adiponectin in postmenopausal women
}

\author{
Su-Jin Kim', Eun-Sun Yoon², Sun-Young Jung ${ }^{3}$, Dae-Young Kim ${ }^{1,4 * *}$ \\ ${ }^{1}$ Institute of Digital Anti-Aging Healthcare, Inje University, Gimhae, Korea \\ ${ }^{2}$ Department of Sports Science, Korea Institute of Sport Science, Seoul, Korea \\ ${ }^{3}$ Department of Physical Therapy, Hosan University, Gyeongsan, Korea \\ ${ }^{4}$ Department of Sports Healthcare, College of Humanities \& Social Sciences, Inje University, Gimhae, Korea
}

Metabolic disorders occurring in menopause, including dyslipidemia, disorders of carbohydrate metabolism, components of metabolic syndrome, constitute risk factors for cardiovascular disease in women. Irisin and fibroblast growth factor-21 (FGF-21), which regulate browning, and high molecular weight (HMW) adiponectin have emerged beneficial for metabolic health. The purpose of the study was to examine the effect of endurance aerobic exercise on circulating browning factor and HMW-adiponectin level in postmenopausal women. Twenty-five postmenopausal women were included in this study. The aerobic exercise program consisted of 60 min of walking exercise at $50 \%-60 \%$ maximum oxygen uptake, and conducted 3 times per week for 6 weeks. Body composition, blood pressure, lipid profiles, physical fitness, and concentration of plasma irisin, FGF-21, HMW-adiponectin were ana- lyzed before and after exercise. Waist circumference, systolic blood pressure, and triglyceride levels decreased, and levels of high-density lipoprotein cholesterol, irisin increased after exercise. However, there were no significant changes in FGF-21 and HMW-adiponectin. Balance and flexibility among physical fitness indices improved after exercise. These results suggested that moderate intensity walking could be the ideal type of exercise in menopausal women to induce a positive change in metabolic health markers, including an increase in irisin levels. Further studies are needed to determine the effects of exercise on brown adipose tissue browning factor.

Keywords: Exercise, Postmenopausal women, Irisin, Fibroblast growth factor-21, High molecular weight adiponectin

\section{INTRODUCTION}

The prevalence of metabolic and cardiovascular diseases, including obesity, rapidly increases due to hormonal changes seen in menopause. As estrogen secretion decreases, total body mass decreases and body fat mass, including abdominal and visceral fat, increases (Sowers et al., 2007). This contributes to the pathogenesis of insulin resistance, and abdominal obesity is a known major risk factor for chronic diseases and increased mortality in postmenopausal women (Folsom et al., 1993). Furthermore, obesity is further accelerated during menopause because of reduced caloric consumption due to decreased total body mass and reduced physical activity. Thus, exercise is essential for the prevention and management of obesity and various chronic diseases in women under- going menopause. The American Heart Association and American College of Sports Medicine (ACSM) recommend at least 150 min of medium-intensity aerobic exercise per week as a part of an exercise program for postmenopausal women (Nelson et al., 2007). According to a review of the effects of exercise in women undergoing menopause, it has been reported that exercise has a variety of health benefits, including the prevention of cardiovascular risk factors, chronic diseases, and cancer, as well as improving mental health (Grindler and Santoro, 2015).

According to recent research trends in obesity and metabolic syndrome, the role of adipokine secreted from adipose tissues and myokine secreted from muscle tissues is highlighted. They affect energy homeostasis, lipid metabolism, inflammation, endothelial cell function, carbohydrate metabolism, insulin sensitivity, and
${ }^{*}$ Corresponding author: Dae-Young Kim (iD https://orcid.org/0000-0002-4662-4463 Department of Sports Health Care, College of Humanities \& Social Sciences, Inje University, 197 Inje-ro, Gimhae 50834, Korea

E-mail: cdps21@inje.ac.kr

Received: April 2, 2020 / Accepted: May 12, 2020
This is an Open Access article distributed under the terms of the Creative Commons Attribution Non-Commercial License (https://creativecommons.org/licenses/by-nc/4.0/) which permits unrestricted non-commercial use, distribution, and reproduction in any medium, provided the original work is properly cited. 
body composition balance (Fasshauer and Blüher, 2015; Li et al., 2017). Adiponectin, a type of adipokine, exists in three subtypes: low molecular weight, medium molecular weight, and high molecular weight (HMW). HMW-adiponectin is known to increase fatty acid oxidation, improve insulin resistance, and has an antiinflammatory function by directly activating the AMP-activated protein kinase (AMPK) present in the liver and skeletal muscles (Hada et al., 2007). Therefore, it is thought that an increase in HMW-adiponectin could aid in the treatment of chronic illnesses. In a previous study in obese middle-aged women, HMW-adiponectin concentration significantly increased and insulin resistance improved after 7 days of high-intensity exercise, and postexercise increase in HMW-adiponectin concentration showed a significant relationship with an increase in the lipid oxidation (Kelly et al., 2012).

Adipose tissues are categorized into white adipose tissue (WAT) and brown adipose tissue (BAT) (Saito, 2014). WAT stores energy in the form of triglycerides (TG), while BAT spends the stored energy (Saely et al., 2012). Recently, brite adipocyte was discovered, which stores energy, similar to WAT, when inactive until activated by a certain stimulus, after which it plays the role of BAT that spends the energy. The activation of this brite adipocyte is referred to as the browning of WAT (Giralt et al., 2013; Lidell et al., 2013; Wu et al., 2012). Furthermore, irisin and fibroblast growth factor-21 (FGF-21), among myokines secreted from muscle tissues, have recently been discussed as factors that cause the browning of the brite adipocyte.

It has been suggested that this process has the metabolic advantage of helping prevent obesity by increasing energy consumption, improving insulin reaction by promoting fatty oxidation, suppressing lipid synthesis, and promoting gluconeogenesis, and increasing the secretion of circulating adiponectin. Cuevas-Ramos et al. (2012) reported that circulating FGF-21 concentration increased after 2 weeks of maximal exercise, and that there was an inverse correlation between FGF-21 concentration and insulin concentration. Furthermore, Boström et al. (2012) reported weight loss and increase in circulating irisin concentration after 10 weeks of walking at an intensity of $65 \%$ of the maximal oxygen uptake in adults. Therefore, it has been shown that promoting or increasing the activation of adiponectin and browning factor can play an important role improving the obesity and metabolic health, and that exercise is a main stimulant of these factors.

Walking is a simple and easily performed exercise, and it is the most common type of exercise performed by adults. Walking can be done without much preparation and by almost anyone as it has low impact on the joints, such as the knees (Myatt et al., 1995). However, walking on a flat surface has a low intensity compared with other exercises, lower level of energy consumption in the same duration, and insufficient loading on the musculoskeletal system. These disadvantages can be overcome by altering the inclination, which consequently changes the angular speed in the knee and ankle joints. The results of walking on a treadmill on $0 \%, 5 \%$, and $10 \%$ inclination showed that the changes in the angle of the joints were the greatest at $10 \%$ inclination. This implies that this would have greater stimulation on the muscular and skeletal system compared with walking on a flat surface. Furthermore, energy metabolism increases with increasing speed and inclination, compared with walking on a flat surface. Therefore, it can be a good alternative exercise method to treat obesity.

However, there is insufficient research into the effect of uphill exercise on browning factor and HMW-adiponectin, and almost no research has been reported on the effect of exercise on adiponectin and browning factor in women undergoing menopause, who are at high risk of obesity and metabolic syndromes. Therefore, this study aimed to identify the effect of medium-intensity aerobic exercise in women undergoing menopause on irisin and FGF-21, as well as concentrations of adiponectin and HMW-adiponectin.

\section{MATERIALS AND METHODS}

\section{Research participants}

A total of 25 women undergoing menopause between the ages of 50 and 70 who have no medical illnesses participated in this study. Women who do not currently smoke and do not have illnesses that would affect their exercise were selected through surveys. In the survey, menopause was defined as at least 1 year having passed since the end of the last menstrual cycle. Those who received surgeries related to the female reproductive system, those who do not know whether they have menopause, those who received hormone replacement therapy, and those with acute or chronic illnesses were all excluded from this study. Before participating in this study, all participants were given sufficient explanations of the contents and the purposes of this research and those who voluntarily wished to participate completed a consent form. The experimental procedure was approved by the Ethics Committee of the Inje University (INJE2019-04-074). The characteristics of the research participants are shown in Table 1.

\section{Exercise program}

In reference to the exercise guidelines of the ACSM (2018), the 
Table 1. Physical characteristics of the subjects $(n=25)$

\begin{tabular}{lr}
\hline Characteristic & \multicolumn{1}{c}{ Mean \pm SD } \\
\hline Age $(\mathrm{yr})$ & $60.28 \pm 5.30$ \\
Height $(\mathrm{cm})$ & $158.17 \pm 5.36$ \\
Weight $(\mathrm{kg})$ & $59.34 \pm 7.80$ \\
Body fat $(\%)$ & $33.62 \pm 4.93$ \\
Body mass index $\left(\mathrm{kg} / \mathrm{m}^{2}\right)$ & $23.72 \pm 2.91$
\end{tabular}

$\mathrm{SD}$, standard deviation.

exercise program in this study comprised three 60 -min ramp courses for walking per week for 6 weeks. The exercise course included an even distribution of uphill, flat, and downhill planes. The mean exercise intensity was medium intensity of about $50 \%-60 \%$ of the maximal heart rate.

\section{Analysis categories and methods}

All measurable variables were measured at the same time using the method before and after exercise in order to analyze the effect of the exercise. To exclude any temporary effect of exercise, postexercise measurements were made $72 \mathrm{hr}$ after the last round of exercise.

\section{Body composition}

As variables of body composition, the height, weight, body fat index, body fat percentage, total body mass, waist circumference, and waist-to-hip ratio (WHR) were measured. Body fat index was calculated by dividing weight $(\mathrm{kg})$ by height $(\mathrm{m})$ squared. Body fat percentage and lean body mass were measured by using a body composition analyzer (Inbody 720, Inbody, Seoul, Korea) that uses a bioelectrical impedance analysis. Waist circumference was measured by taking the area between the lowest part of the ribs and the iliac crest with a measuring tape. Hip circumference was measured by taking the widest area of the hip to calculate the WHR.

\section{Blood testing}

Blood tests were performed on all subjects after $12 \mathrm{hr}$ of fasting. Blood was drawn at the same time under the same conditions before and after exercise. Five milliliters of venous blood were drawn from the anterior cardinal vein using a disposable needle, stored in an serum separation tube for serum and centrifuged for $10 \mathrm{~min}$ at 3,000 rpm; $200 \mu \mathrm{L}$ of the serum was stored in a sample tube, and a reagent strip was attached to analyze the total cholesterol (TC), TG, and high-density lipoprotein cholesterol (HDL-C) using an automated biochemical analyzer (Spotechem Ez SP-4430, Arkray, Tokyo, Japan). LDL-C was calculated using the Friedewald for- mula: TC - HDL-C - (TG/5). Blood glucose was analyzed using a blood glucose meter (Accuchek, Roche, Germany) by putting $0.6 \mu \mathrm{L}$ of the whole blood drawn from each subject's finger on the test kit. Glycated hemoglobin was analyzed using the glycated hemoglobin test machine (Easy A1C, Infopia, Seoul, Korea); $4 \mu \mathrm{L}$ of the whole blood drawn from each subject's vein was placed into the cartridge and inserted into the test machine for analysis.

Irisin and FGF-21 were analyzed with the enzyme-linked immunosorbent assay (ELISA) method, using the Irisin ELISA kit (BioVendor, Brno, Czech) and the FGF-21 Quantikine ELISA kit (R\&D System, Minneapolis, MN, USA). Adiponectin and HMWadiponectin were analyzed with the ELISA method, using the Adiponectin Human ELISA kit (BioVendorh) and the Human HMWadiponectin/Acrp30 Quantikine ELISA kit (R\&D System).

\section{Blood pressure}

After 10 min of rest in a sitting position, blood pressure was measured in the right arm using an automatic blood pressure gauge (Bpbio320, Inbody). Measurements were taken twice, 3 min apart and the mean values were used for data processing. The mean arterial pressure was calculated with the following formula: mean arterial pressure $=$ diastolic blood pressure + (systolic blood pressure - diastolic blood pressure/3).

\section{Physical fitness measurement}

To measure the maximum muscular strength, each subject held the digital dynamometer (DW-781, Daewoosports Industry, Seoul, Korea) with all fingers excluding the thumb, and adjusted the width so that the second joint formed a right angle. Subjects relaxed their arm and put maximum force for 5 sec while being careful not to have the dynamometer touch their body to make the measurement. The greater value of two measurements was recorded in increments of $0.1 \mathrm{~kg}$. To measure muscular endurance, subjects performed sit-ups using a sit-up board (NXF 602, Kaesunsports, Seoul, Korea). Each subject supported the head with their hands, and lifted the body until the elbows touched the knees and lowered the body until the shoulders touched the sit-up board. The subjects were instructed not to take advantage of a rebounding effect. The number of sit-ups done in a minute was measured. To measure flexibility, a sit-and-reach measurement system was used. Each bare-footed subject sits down straight with her knees straightened while placing the bottom of her feet against the footstall. The distance between the two straightened legs does not exceed $5 \mathrm{~cm}$. Then, the subject puts two hands together, and pushes the measurement bar as far as possible and maintains this posture 
for $3 \mathrm{sec}$. This distance was recorded. Bending the knees or hitting the measurement bar using rebounding was not permitted. Two measurements were taken in increments of $0.1 \mathrm{~cm}$, and the better measurement was recorded. To measure balance, each subject lifted their hands to shoulder height and with eyes closed, lifted the foot of their choice and maintained this posture for as long as possible. The duration was measured twice and the better record was selected.

\section{Data processing}

All measured data were represented in means and standard deviations. To analyze the effect of the exercises, a paired sample $t$-test was performed on the pre- and postexercise values. All statistical processing was performed with IBM SPSS Statistics ver. 25.0 (IBM Co., Armonk, NY, USA), and the significance level $(\alpha)$ was set at $P \leq 0.05$.

\section{RESULTS}

\section{Change in physique and body composition}

The changes in the body composition after uphill walking are shown in Table 2. Compared with the status before the exercise, waist circumferences decreased significantly by about $1 \mathrm{~cm}(P=$ 0.039), and there were no changes in weight, body fat index, or

Table 2. Changes in the obesity indexes after 6 weeks of exercise training

\begin{tabular}{lcccc}
\hline Variable & Pre & Post & $\Delta$ score & $P$-value \\
\hline Weight $(\mathrm{kg})$ & $59.34 \pm 7.80$ & $59.65 \pm 7.55$ & $0.31 \pm 0.80$ & 0.064 \\
BMI $\left(\mathrm{kg} / \mathrm{m}^{2}\right)$ & $23.72 \pm 2.91$ & $23.85 \pm 2.81$ & $0.12 \pm 0.32$ & 0.062 \\
Body fat $(\%)$ & $33.62 \pm 4.93$ & $33.31 \pm 5.30$ & $-0.31 \pm 1.73$ & 0.377 \\
LBM $(\mathrm{kg})$ & $21.15 \pm 2.57$ & $21.39 \pm 2.69$ & $0.24 \pm 0.58$ & 0.053 \\
WC $(\mathrm{cm})$ & $83.32 \pm 7.32$ & $82.30 \pm 8.18$ & $-1.01 \pm 2.32$ & 0.039 \\
\hline
\end{tabular}

Values are presented as mean \pm standard deviation.

$\Delta$ score represents the difference in score after 6 weeks from the score before the exercise program.

BMI, body mass index; LBM, lean body mass; WC, waist circumference. body fat percentage. The increase in total body mass was not statistically significant $(P=0.053)$.

\section{Change in metabolic risk factors}

The changes in metabolic health indices after uphill walking are shown in Table 3. Systolic blood pressure decreased significantly by about $5.6 \%(P=0.020)$, TC by $8.1 \%(P<0.001)$, TG by $35.9 \%(P=0.017)$, and LDL-C by $12.8 \%(P=0.001)$, while HDL-C significantly increased by $6.3 \%(P=0.016)$. However, there were no statistically significant differences in fasting blood glucose and glycated hemoglobin levels.

\section{Changes in irisin, FGF-21, adiponectin, HMW-adiponectin}

Changes in irisin, FGF-21, adiponectin, and HMW-adiponectin after uphill walking are shown in Table 4. Irisin significantly increased by about $20.5 \%(P=0.007)$, while FGF-21, adiponectin and HMW-adiponectin decreased but did not show a statistically significant difference $(P=0.150)$.

\section{Changes in health-related physical fitness}

Table 3. Changes in the metabolic risk factors after 6 weeks of exercise training

\begin{tabular}{lcccc}
\hline Variable & Pre & Post & $\Delta$ score & $P$-value \\
\hline SBP $(\mathrm{mmHg})$ & $127.76 \pm 14.60$ & $120.64 \pm 10.96$ & $-7.12 \pm 14.25$ & 0.020 \\
DBP $(\mathrm{mmHg})$ & $76.44 \pm 17.72$ & $73.12 \pm 9.88$ & $-3.32 \pm 17.61$ & 0.355 \\
Glucose $(\mathrm{mg} / \mathrm{dL})$ & $92.36 \pm 11.88$ & $93.84 \pm 11.59$ & $1.48 \pm 9.55$ & 0.446 \\
HbA1c $(\%)$ & $5.41 \pm 0.66$ & $5.31 \pm 0.73$ & $-0.10 \pm 0.34$ & 0.158 \\
TC $(\mathrm{mg} / \mathrm{dL})$ & $187.92 \pm 34.92$ & $172.72 \pm 29.86$ & $-15.20 \pm 16.53$ & 0.001 \\
TG $(\mathrm{mg} / \mathrm{dL})$ & $112.44 \pm 84.74$ & $72.08 \pm 30.89$ & $-40.36 \pm 78.70$ & 0.017 \\
LDLC $(\mathrm{mg} / \mathrm{dL})$ & $106.19 \pm 31.04$ & $92.63 \pm 28.27$ & $-10.96 \pm 20.63$ & 0.001 \\
HDLC $(\mathrm{mg} / \mathrm{dL})$ & $60.56 \pm 13.02$ & $64.40 \pm 11.64$ & $3.84 \pm 7.40$ & 0.016 \\
\hline
\end{tabular}

Values are presented as mean \pm standard deviation.

$\Delta$ score represents the difference in score after 6 weeks from the score before the exercise program.

SBP, systolic blood pressure; DBP, diastolic blood pressure; HbA1c, hemoglobin A1c; TC, total cholesterol; TG, triglycerides; LDLC, low-density lipoprotein cholesterol; HDLC, high-density lipoprotein cholesterol.

Table 4. Changes in browning factor, adiponectin, HMW-adiponectin

\begin{tabular}{|c|c|c|c|c|}
\hline Variable & Pre & Post & $\Delta$ score & $P$-value \\
\hline $\operatorname{lrisin}(\mu \mathrm{g} / \mathrm{mL})$ & $2.70 \pm 1.13$ & $3.08 \pm 1.11$ & $0.37 \pm 0.64$ & 0.007 \\
\hline FGF-21 (pg/mL) & $216.87 \pm 119.15$ & $186.08 \pm 105.70$ & $-30.79 \pm 110.52$ & 0.176 \\
\hline Adiponectin (mg/dL) & $10.02 \pm 5.05$ & $9.58 \pm 4.33$ & $-0.44 \pm 2.12$ & 0.395 \\
\hline HMW-adiponectin (ng/mL) & $3,511.67 \pm 2,226.37$ & $3,288.30 \pm 2,183.52$ & $-623.36 \pm 1,174.62$ & 0.309 \\
\hline
\end{tabular}

Values are presented as mean \pm standard deviation.

$\Delta$ score represents the difference in score after 6 weeks from the score before the exercise program.

HMW, high molecular weight; FGF-21, fibroblast growth factor-21. 
Table 5. Changes in health-related physical fitness

\begin{tabular}{lllcc}
\hline Variable & \multicolumn{1}{c}{ Pre } & \multicolumn{1}{c}{ Post } & $\Delta$ score & $P$-value \\
\hline Muscle strength (kg) & $37.91 \pm 14.86$ & $36.12 \pm 12.77$ & $-1.78 \pm 5.65$ & 0.259 \\
Muscle endurance $($ reps) & $16.16 \pm 10.85$ & $19.56 \pm 11.89$ & $3.40 \pm 8.74$ & 0.064 \\
Flexibility $(\mathrm{cm})$ & $17.09 \pm 7.24$ & $19.59 \pm 5.90$ & $2.50 \pm 3.73$ & 0.003 \\
Balance $(\mathrm{sec})$ & $10.05 \pm 6.99$ & $21.63 \pm 21.03$ & $11.58 \pm 21.13$ & 0.011
\end{tabular}

Values are presented as mean \pm standard deviation.

$\Delta$ score represents the difference in score after 6 weeks from the score before the exercise program.

Changes in physical fitness after uphill walking are shown in Table 5. Flexibility increased significantly by $14 \%(P=0.003)$. Balance also increased significantly by $115 \%(P=0.011)$. Strength and muscular endurance both showed a trend towards increasing, but there were no statistically significant differences.

\section{DISCUSSION}

This study aimed to examine the effect of uphill walking on energy consumption, lipid metabolism, irisin, and FGF-21, which are browning factors, and adiponectin in healthy women undergoing menopause. The results showed that there were no significant changes in weight or adiponectin after exercise, but irisin significantly increased and metabolic health indices improved.

BAT, which transforms energy to heat-generating energy and stores it, was discovered in adults through imaging technology. With this discovery, there is a growing interest in research focused on the prevention of metabolic syndromes, including browning hormones and obesity (Kajimura and Saito, 2014). Irisin stimulates the browning of the WAT and the expression of uncoupling protein 1 , thus increasing the energy consumption and activating lipid and glucose metabolism to combat obesity and metabolic syndrome. Furthermore, its secretion increases when the muscle contracts, suggesting that exercise plays an important role in increasing the level of irisin. According to research on the link between exercise and irisin reported to date, the results differ depending on the type of exercise but aerobic exercise increases the concentration of circulating irisin (Crujeiras et al., 2015; Stengel et al., 2013). This was consistent with previous findings, showing increased circulating irisin concentration and decreased waist circumference, systolic blood pressure, and circulating lipid after 6 weeks of medium-intensity exercise in women undergoing menopause.

FGF-21 is reported to improve the insulin response by promoting the lipid oxidation in the liver and promoting ketogenesis and gluconeogenesis. Furthermore, FGF-21 promotes the production of peroxisome proliferator-activated receptor (PPAR)- $\gamma$ and adiponectin in WAT, thereby secreting adiponectin to the blood and providing a metabolic advantage of promoting heat generation in BAT (Reitman, 2007). Aerobic exercise is thought to increase the expression of FGF-21 by activating PPAR- $\alpha$, but the effect of exercise in FGF-21 is not as clear in research to date.

In this study, FGF-21 concentration tended to decrease, but not significantly, after 6 weeks of walking. This is in line with findings by Taniguchi et al. (2016) who reported that there were decrease in serum FGF-21 concentration in Japanese elderly males after 3 cycle exercises per week for 5 weeks. In contrast, Catoire et al. (2014) found increased expression of FGF-21 in the muscles after $1 \mathrm{hr}$ of cycling. Significant increases after 2 weeks of exercise have been reported (Cuevas-Ramos et al., 2012) and after 1-time exercise (Kim et al., 2013), as well as findings showing exercise having no effect. Thus, the effect of FGF-21 has not yet been clearly established. According to Cuevas-Ramos et al. (2012), there is a negative correlation between FGF-21 and insulin, and the possibility that increased insulin due to exercise suppresses the secretion of FGF-21. Although the subjects of this study all showed normal blood glucose levels, there is a positive correlation between insulin resistance measured with TG/HDL-C and FGF-21. Therefore, research on the changes in FGF-21 concentration with a focus on the type of exercise and insulin concentration will be needed in the future.

HMW-adiponectin, which is one of the subtypes of adiponectin, has been shown to directly activate AMPK in the liver and the musculoskeletal system and increase the fatty acid oxidation to improve insulin resistance and also has anti-inflammatory functions (Hada et al., 2007). Additionally, the increase in adiponectin after exercise has been shown to improve metabolic syndrome and cardiovascular diseases. Kim et al. (2014) reported an increase in HMW-adiponectin in obese middle-aged women after 24 weeks of regular exercise, a positive correlation with total-adiponectin, and negative correlations with glucose and free fatty acid. However, this study showed a trending decrease in adiponectin and HMWadiponectin after exercises, in contrast with previous findings. It has been suggested that at least $5 \%$ weight loss is necessary to increase adiponectin (Varady et al., 2009), and that HMW-adiponectin can increase significantly through weight loss due to high-intensity aerobic training (Garekani et al., 2011) and diet control (Hulver et al., 2002). Studies showing no significant changes in adiponectin after exercise also showed an insignificant weight loss effect (Hulver et al., 2002). Nevertheless, it is thought that weight 
loss through a relatively high-intensity exercise should accompany an increase in adiponectin.

Among health-related physical fitness factors, significant increases were observed in balance and flexibility. Similar findings have demonstrated that walking improved functional physical fitness factors, including flexibility, in middle-aged women (Kim, 2018). The increase in flexibility may be due to the increase range of motion of the hip joints caused by the highly sloped mountain terrain, unlike flat surfaces and thus leading to natural contraction and relaxation of the gastrocnemius muscle and the popliteal muscle above the knee. The increase in balance can be considered to be due to the development of sensory-motor neurons for motion and balancing in multidimensional situations that result from irregular mountain terrains.

Interestingly, this study found that irisin and all circulating lipid profiles significantly improved despite the short duration of exercise. According to the results from the meta-analysis on the improvement of blood lipid profile from exercise, blood lipid improvements such as an increase in HDL-C and a decrease in TG and LDL-C occur after at least 12 weeks of aerobic exercise. Improvement in blood lipid profile is more apparent with increasing intensity of exercise (Mann et al., 2014). However, greater improvement on the blood lipid profile was seen in our study even with medium-intensity duration of exercise for 6 weeks, than in the meta-analysis. Irisin also showed significant increases through endurance training rather than resistance training and through high-intensity training rather than low-intensity training (Daskalopoulou et al., 2014; Tsuchiya et al., 2014). However, in this study, the expression of irisin was sufficiently increased in women undergoing menopause with only medium-intensity uphill walking exercises. These results provide evidence that a short duration of medium-intensity uphill walking exercise can improve lipid profiles and increase the browning factor in women undergoing menopause who are at high risk of exposure to various metabolic syndromes and cardiovascular diseases due to increased body fat and reduced muscle mass, and also that the uphill walking is more effective in treating obesity compared with walking on a flat surface.

\section{CONFLICT OF INTEREST}

No potential conflict of interest relevant to this article was reported.

\section{ACKNOWLEDGMENTS}

This work was supported by the 2018 Inje University research grant.

\section{REFERENCES}

American College of Sports Medicine. ACSM's Guidelines for exercise testing and prescription. 10th ed. Philadelphia (PA): Lippincott Williams \& Wilkins, 2018.

Boström P, Wu J, Jedrychowski MP, Korde A, Ye L, Lo JC, Rasbach KA, Boström EA, Choi JH, Long JZ, Kajimura S, Zingaretti MC, Vind BF, Tu H, Cinti S, Højlund K, Gygi SP, Spiegelman BM. A PGC1- $\alpha$-dependent myokine that drives brown-fat-like development of white fat and thermogenesis. Nature 2012;481:463-468.

Catoire M, Mensink M, Kalkhoven E, Schrauwen P, Kersten S. Identification of human exercise-induced myokines using secretome analysis. Physiol Genomics 2014;46:256-267.

Crujeiras AB, Pardo M, Casanueva FF. Irisin: 'fat' or artefact. Clin Endocrinol (Oxf) 2015;82:467-474.

Cuevas-Ramos D, Almeda-Valdés P, Meza-Arana CE, Brito-Córdova G, Gómez-Pérez FJ, Mehta R, Oseguera-Moguel J, Aguilar-Salinas CA. Exercise increases serum fibroblast growth factor 21 (FGF21) levels. PLoS One 2012;7:e38022.

Daskalopoulou SS, Cooke AB, Gomez YH, Mutter AF, Filippaios A, Mesfum ET, Mantzoros CS. Plasma irisin levels progressively increase in response to increasing exercise workloads in young, healthy, active subjects. Eur J Endocrinol 2014;171:343-352.

Fasshauer M, Blüher M. Adipokines in health and disease. Trends Pharmacol Sci 2015;36:461-470.

Folsom AR, Kaye SA, Sellers TA, Hong CP, Cerhan JR, Potter JD, Prineas RJ. Body fat distribution and 5-year risk of death in older women. JAMA 1993;269:483-487.

Garekani ET, Mohebbi H, Kraemer RR, Fathi R. Exercise training intensity/volume affects plasma and tissue adiponectin concentrations in the male rat. Peptides 2011;32:1008-1012.

Giralt M, Villarroya F. White, brown, beige/brite: different adipose cells for different functions? Endocrinology 2013;154:2992-3000.

Grindler NM, Santoro NF. Menopause and exercise. Menopause 2015;22: 1351-1358.

Hada Y, Yamauchi T, Waki H, Tsuchida A, Hara K, Yago H, Miyazaki O, Ebinuma $\mathrm{H}$, Kadowaki T. Selective purification and characterization of adiponectin multimer species from human plasma. Biochem Biophys Res Commun 2007;356:487-493.

Hulver MW, Zheng D, Tanner CJ, Houmard JA, Kraus WE, Slentz CA, 
Sinha MK, Pories WJ, MacDonald KG, Dohm GL. Adiponectin is not altered with exercise training despite enhanced insulin action. Am J Physiol Endocrinol Metab 2002;283:E861-E865.

Kajimura S, Saito M. A new era in brown adipose tissue biology: molecular control of brown fat development and energy homeostasis. Annu Rev Physiol 2014;76:225-249.

Kelly KR, Blaszczak A, Haus JM, Patrick-Melin A, Fealy CE, Solomon TP, Kalinski MI, Kirwan JP. A 7-d exercise program increases high-molecular weight adiponectin in obese adults. Med Sci Sports Exerc 2012;44: 69-74.

Kim DY. Effects of exercise using a mobile device on cardiopulmonary function, metabolic risk factors, and self-efficacy in obese women. J Exerc Rehabil 2018;14:829-834.

Kim DY, Seo BD, Kim DJ. Effect of walking exercise on changes in cardiorespiratory fitness, metabolic syndrome markers, and high-molecularweight adiponectin in obese middle-aged women. J Phys Ther Sci 2014;26:1723-1727.

Kim KH, Kim SH, Min YK, Yang HM, Lee JB, Lee MS. Acute exercise induces FGF21 expression in mice and in healthy humans. PLoS One 2013;8:e63517.

Li F, Li Y, Duan Y, Hu CA, Tang Y, Yin Y. Myokines and adipokines: involvement in the crosstalk between skeletal muscle and adipose tissue. Cytokine Growth Factor Rev 2017;33:73-82.

Lidell ME, Betz MJ, Dahlqvist Leinhard O, Heglind M, Elander L, Slawik M, Mussack T, Nilsson D, Romu T, Nuutila P, Virtanen KA, Beuschlein F, Persson A, Borga M, Enerbäck S. Evidence for two types of brown adipose tissue in humans. Nat Med 2013;19:631-634.

Mann S, Beedie C, Jimenez A. Differential effects of aerobic exercise, resistance training and combined exercise modalities on cholesterol and the lipid profile: review, synthesis and recommendations. Sports Med 2014;44:211-221.

Myatt G, Baxter R, Dougherty R, Williams G, Halle J, Stetts D, Underwood F. The cardiopulmonary cost of backward walking at selected speeds. J Orthop Sports Phys Ther 1995;21:132-138.
Nelson ME, Rejeski WJ, Blair SN, Duncan PW, Judge JO, King AC, Macera CA, Castaneda-Sceppa C. Physical activity and public health in older adults: recommendation from the American College of Sports Medicine and the American Heart Association. Med Sci Sports Exerc 2007;39:1435-1445.

Reitman ML. FGF21: a missing link in the biology of fasting. Cell Metab 2007;5:405-407.

Saely CH, Geiger K, Drexel H. Brown versus white adipose tissue: a minireview. Gerontology 2012;58:15-23.

Saito M. Human brown adipose tissue: regulation and anti-obesity potential. Endocr J 2014;61:409-416.

Sowers M, Tomey K, Jannausch M, Eyvazzadeh A, Nan B, Randolph J Jr. Physical functioning and menopause states. Obstet Gynecol 2007;110: 1290-1296.

Stengel A, Hofmann T, Goebel-Stengel M, Elbelt U, Kobelt P, Klapp BF. Circulating levels of irisin in patients with anorexia nervosa and different stages of obesity--correlation with body mass index. Peptides 2013;39:125-130.

Taniguchi H, Tanisawa K, Sun X, Kubo T, Higuchi M. Endurance exercise reduces hepatic fat content and serum fibroblast growth factor 21 levels in elderly men. J Clin Endocrinol Metab 2016;101:191-198.

Tsuchiya Y, Ando D, Goto K, Kiuchi M, Yamakita M, Koyama K. High-intensity exercise causes greater irisin response compared with low-intensity exercise under similar energy consumption. Tohoku J Exp Med 2014;233:135-140.

Varady KA, Tussing L, Bhutani S, Braunschweig CL. Degree of weight loss required to improve adipokine concentrations and decrease fat cell size in severely obese women. Metabolism 2009;58:1096-1101.

Wu J, Boström P, Sparks LM, Ye L, Choi JH, Giang AH, Khandekar M, Virtanen KA, Nuutila P, Schaart G, Huang K, Tu H, van Marken Lichtenbelt WD, Hoeks J, Enerbäck S, Schrauwen P, Spiegelman BM. Beige adipocytes are a distinct type of thermogenic fat cell in mouse and human. Cell 2012;150:366-376. 